\title{
Copper Particles Generated During in situ Laser-induced Synthesis Exhibit Catalytic Activity Towards Formation of Gas Phase
}

\author{
Dmitrii Gordeychuk*, Vladimir Kochemirovsky*, Victor Sorokoumov*, Ilya Tumkin*, Alexey Kuzmin ${ }^{* *}$ and Irina Balova* \\ *Saint Petersburg State University, 7/9 Universitetskaya nab., St. Petersburg, 199034 Russia. \\ E-mail: lasergroupspb@gmail.com \\ ** Institute for Analytical Instrumentation of the Russian Academy of Sciences, 198095,Saint- \\ Petersburg, ulitsa Ivana Chernykh, 31-33, lit. A, Russia.
}

\begin{abstract}
The method of laser-induced chemical deposition of metal from solution was applied in the continuous in situ laser generation of metal copper catalysts for model organic synthesis reactions. The gas phase products producing during laser-induced copper deposition were analyzed by gas mass spectroscopy whereas solutions used for the copper deposition were investigated before and after laser irradiation using chromato-mass and NMR spectroscopy. It was found out that the catalysis of the studied organic reactions by metal catalysts generated during laser deposition process occurs only upon laser irradiation of the reaction mixture, in turn, the copper structures deposited under laser light exhibit no catalytic activity.
\end{abstract}

DOI: 10.2961/jlmn.2017.02.0001

Keywords: laser-induced metal deposition, catalysis, copper, gas mass spectroscopy

\section{Introduction.}

One of the most important scientific and engineering tasks is the development of new types of catalysts for selective organic synthesis. Among them are easily renewable and adaptable for flow-through reactors heterogeneous catalysts which provide advantages compared to homogeneous catalysis. It is known that the nanostructured and highly porous materials with a large specific surface area exhibit the highest catalytic activity. Therefore, the development of novel catalytic systems for the organic reactions catalyzed by metal nanosructures is an actual problem of modern chemistry. As a rule, for that purpose the expensive metals such as gold, platinum, palladium, etc are used. On the other hand, the use of cheaper metals such as copper, cobalt, zinc, etc results in fast "contamination" of the catalyst surface by reaction products and the loss of activity. In addition, regeneration of waste catalysts and the synthesis of new is time consuming and quite expensive process.

The technique used in this work provides the in situ catalyst generation and in which the problem with activation of the catalyst surface is diminished by constant replacement of the contaminated during the generation process catalyst by new one. In this approach, nano-sized metal particles generated upon laser irradiation are involved in the catalysis process then these particles are deposited forming the pure metal structures suitable for further usage or regeneration. Here, we propose to use of laser induced chemical liquid-phase deposition (LCLD) for the aforementioned purposes. In general, LCLD is a technique dealing with a treatment of a local region of the dielectric substrate or conductor on air or under a layer of liquid solution (plating solution) by a focused laser beam [1-4, 10-12]. In turn, laser irradiation of the plating solution leads to the acceleration of metal reduction reaction in the irradiated area as a result of the temperature increase in the local volume of solution within the focus of the laser beam. LCLD can be utilized for deposition of many metals [4-10] and can find applications in microelectronics as well as in the manufacturing of micro-sensors. Furthermore, the versatility of the LCLD method is that the chemical and physical parameters for the efficient metal deposition can be adjusted to any substrate surface or the reaction mixture. There are many factors which affect the deposition process such as the influence of the substrate type, the concentration of platting solution, laser power etc., however, the influence of reactions occur in solution as well as the intensive formation of the gaseous products upon laser irradiation was either ignored or poorly studied so far [4]. Indeed it was only recently figured out that gas-phase reactions and reactions in solution may be caused by the catalytic activity of nanophase nucleates formed in the very first step of the process of laser-induced chemical deposition of metal. It is clear that further progress in this area is possible only on the basis of deep research of the chemical mechanisms of laser-induced reactions and mechanisms of heterogeneous catalysis occurred under laser radiation. Thus, in this paper we discuss the catalytic properties of copper structures which form during the laser-induced deposition as well as their influence on the processes occurred in solution and their activity in the proposed model reactions.

\section{Experimental.}

All chemicals used in this work were of analytical grade and purchased commercially (Sigma Aldrich). The detailed description of the experimental setup for the laserinduced metal deposition has been published elsewhere $[11,13]$. Briefly, the from a continuous wave $532 \mathrm{~nm}$ diodepumped solid-state Nd:YAG laser is split into two parts. The first part is focused on the boundary region between metal salt solution and dielectric substrate placed in the cell. This cell is mounted on the computer controlled motorized stage used for adjustment of the focal point position where the deposition reaction starts. The second part is sent to web-camera used for in situ monitoring and control the 
laser-induced synthesis. The LCLD experiments were conducted at the scanning speed of $2.5 \mu \mathrm{m} \mathrm{s}^{-1}$. Profile of the laser beam had Gaussian distribution with circular shape, the diameter of the focal spot was 7 microns. Glassceramics (Sitall ST-50-1) and common $\mathrm{SiO}_{2}$ based glass were used as dielectric substrates. Sitall ST-50-1 is wellknown and widely used in microelectronics material, which is commercially available and composed of $\mathrm{SiO}_{2}(60.5 \%)$, $\mathrm{Al}_{2} \mathrm{O}_{3}$ (13.5\%), $\mathrm{CaO}$ (8.5\%), $\mathrm{MgO}$ (7.5\%) and $\mathrm{TiO}_{2}$ $(10.0 \%)$. In turn, the glass substrate is composed of $\mathrm{SiO}_{2}$ (75\%), $\mathrm{K}_{2} \mathrm{O}+\mathrm{Na}_{2} \mathrm{O}$ (25\%). Copper lines were deposited on the surfaces of glass-ceramics and glass at laser power of 900 and $650 \mathrm{~mW}$, respectively.

Optical images of copper structures were obtained using an optical microscope with $40 \times$ magnification (MMN-2, LOMO). The topology of the synthesized copper deposits was observed by means of scanning electron microscopy (SEM). The atomic composition of these deposits was studied using energy dispersion of X-ray spectroscopy (EDX). The EDX-system was coupled with a Zeiss Supra $40 \mathrm{VP}$ scanning electron microscope equipped with X-ray attachment (Oxford Instruments INCA X-act). The electrical conductivity properties of the deposited copper structures were studied with an impedance meter Z-2000 (Elins Co.) in the frequency band of $20 \mathrm{~Hz}$ to $2 \mathrm{MHz}$ at the signal amplitude of $125 \mathrm{mV}$.

A Bruker $400 \mathrm{MHz}$ WB Avance III NMR spectrometer was used to record H-NMR spectra of solutions used for the copper deposition before and after laser irradiation.

A portable quadrupole gas mass spectrometer (MS7100) was used to obtain mass spectra and the time dependencies of intensities of gas phase products producing during laser-induced chemical deposition of copper.

The analysis of solutions used for the copper deposition after laser irradiation was carried out using chromato-massspectrometer GCMS-QP2010 Ultra equipped with a combined dispenser (AOC-5000) for automatic dispensing of liquid samples and samples of the equilibrium vapor.

\section{Results and Discussion.}

In order to find the optimum conditions for in situ laserinduced synthesis the laser metal chemical deposition of copper experiments were performed for two different solutions and dielectric substrates. The solution compositions and types of substrates used in these experiments are shown in Table 1.

Table 1 The compositions of solutions used for laser-induced chemical deposition of copper on glass-ceramics Sitall (1) and glass (2).

\begin{tabular}{ccccc}
\hline & $\begin{array}{c}\mathrm{CuCl}_{2} \\
\mathrm{~mol} \mathrm{~L}^{-1}\end{array}$ & $\begin{array}{c}\mathrm{C}_{10} \mathrm{H}_{16} \mathrm{~N}_{2} \mathrm{O}_{8} \\
\mathrm{~mol} \mathrm{~L}^{-1}\end{array}$ & $\begin{array}{c}\mathrm{KNaC}_{4} \mathrm{H}_{4} \mathrm{O}_{6} \times 4 \mathrm{H}_{2} \mathrm{O} \\
\mathrm{mol} \mathrm{L}^{-1}\end{array}$ & $\begin{array}{c}\mathrm{NaOH} \\
\mathrm{mol} \mathrm{L}^{-1}\end{array}$ \\
\hline $\mathbf{1}$ & 0.01 & - & 0.033 & 0.1 \\
$\mathbf{2}$ & 0.01 & 0.011 & - & 0.1 \\
\hline
\end{tabular}

In both experiments we used a component (EDTA and sodium potassium tartrate) combining the functions of reducing agent and ligand. As a result, the copper lines of about a $100 \mu \mathrm{m}$ wide, $6 \mu \mathrm{m}$ thick and $1 \mathrm{~cm}$ long were produced using the LCLD technique. Fig. 1 (a, c) demonstrates SEM images of typical copper microstructures deposited on glass and glass-ceramics. The results of scanning elec- tron microscopy show that the deposited copper structures have a highly developed surface. In turn, the study of the elemental composition these deposits using the EDX (Fig. 1 (b, d)) reveals that copper is the primary component. Continuous copper line with length of $1 \mathrm{~cm}$, width of 50$100 \mu \mathrm{m}$ and thickness of about $6 \mu \mathrm{m}$ has an electrical resistance of 1.5-3 $\Omega$, which is 3-6 times higher than those exhibited by pure monolithic copper. The thickness of the copper line was measured using ion etching technique. Thus, the implementation of the aforementioned solutions in LCLD experiments allowed to produce copper structures with good topology and high electrical conductivity properties, as a result, the compositions of these solutions were chosen for further studies.
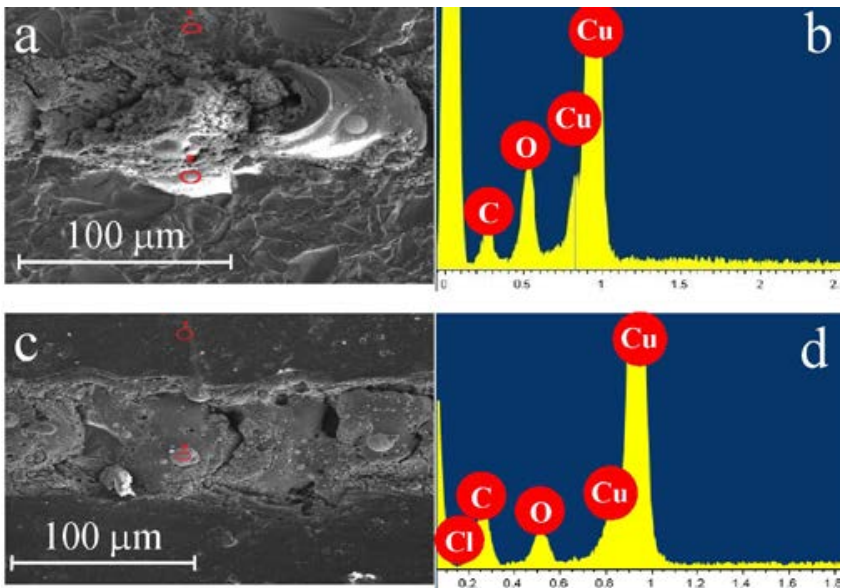

Fig. 1 SEM image (a) and EDX spectrum (b) of the copper line deposited from solution 1 on glass-ceramics Sitall , SEM image (c) and EDX spectrum (d) of the copper line deposited from solution 2 on glass.

It is known that the LCLD mechanism is still under debate and not completely clear [1-5]. In order to shed light on this problem one should know how metal is reduced during the laser-induced chemical deposition process. Of course, it is very challenging task which requires different approaches and in this work we tried to consider only few aspects which eventually will help us to solve this problem. For that purpose we performed the series of experiments focused on the identification of the gas phase which forms during LCLD process and analysis of solutions used for the copper deposition before and after laser irradiation.

The gas phase products were collected directly during laser deposition reaction. In these experiments we used glass and glass-ceramics because upon laser irradiation these materials do not produce any decomposition products which can potentially affect the studied gas phase. Also, we applied the solutions described above which were previously successfully used for copper deposition and exhibit high gas formation capability upon laser irradiation. On the other hand, the compositions of these solutions are simple and consisted of only three components which is better for understanding of their influence on the gas phase generation process. Finally, in order to identify gas phase composition we decided to apply gas mass spectrometry. All experiments were conducted on our LCLD set up using standard procedure. Here, we used modified cell which was connected to mass spectrometer. As a result, we were able to 
fix the composition of gas phase under the solution surface before initiation of the deposition reaction and control it during the reaction progress.

The gas mass spectroscopic studies revealed the release of the unsaturated hydrocarbons during LCLD process. It is should be noted that the formation of such products cannot be explained by the classical reactions previously used for description of the LCLD mechanism. Moreover, these products can be formed only in the presence of catalysts, including copper, thus, the catalytic nature of this process has been proposed (Fig. 2).

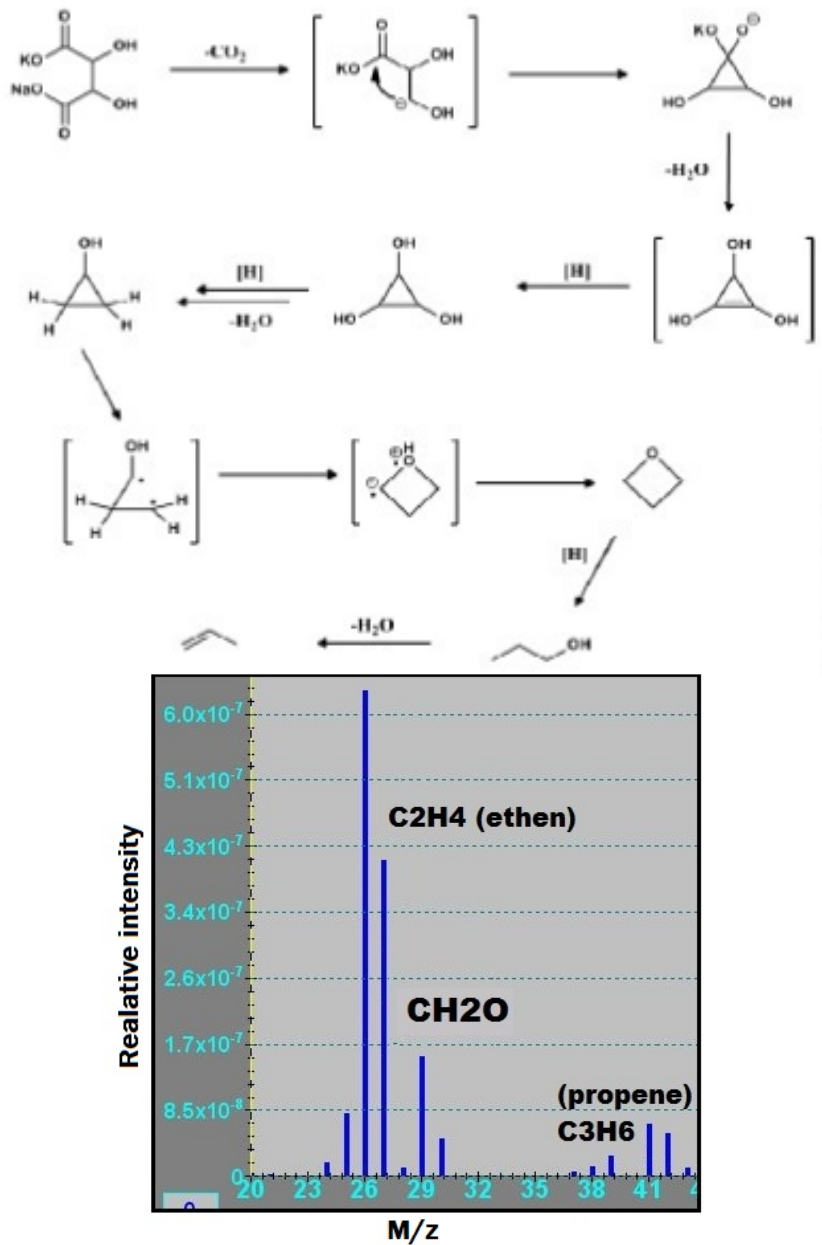

Fig. 2 The scheme of possible catalytic reaction that can occur within the focus of the laser beam during the LCLD process. Gas mass spectra of gas phase products produced during laser-induced chemical deposition process from solution 1 .

A study of gas mass spectra of individual solution components showed that the generation of the gas phase products in the focus of the laser beam is possible only in the presence of copper salt. We believe that there are two possible ways of formation of active catalysts during laserinduced deposition process: stationary and dynamic. In the stationary regime, the catalysis occurs on already formed copper deposits. On the other hand, in the dynamic mode the catalysis occurs during the formation and active growth phase of nucleate directly upon laser irradiation.

The main components of gas phase products released during laser-induced deposition process for system $\mathrm{CuCl}_{2}$ EDTA and $\mathrm{CuCl}_{2}$-tartrate were identified. For all studied solutions these components correspond to hydrogen, eth- ylene and carbon dioxide. Based on the obtained concentration dependences for the studied solutions, it was shown that the increase of the ligand concentration results in the increase of the yield of the main components of the gas phase products (Fig. 3).

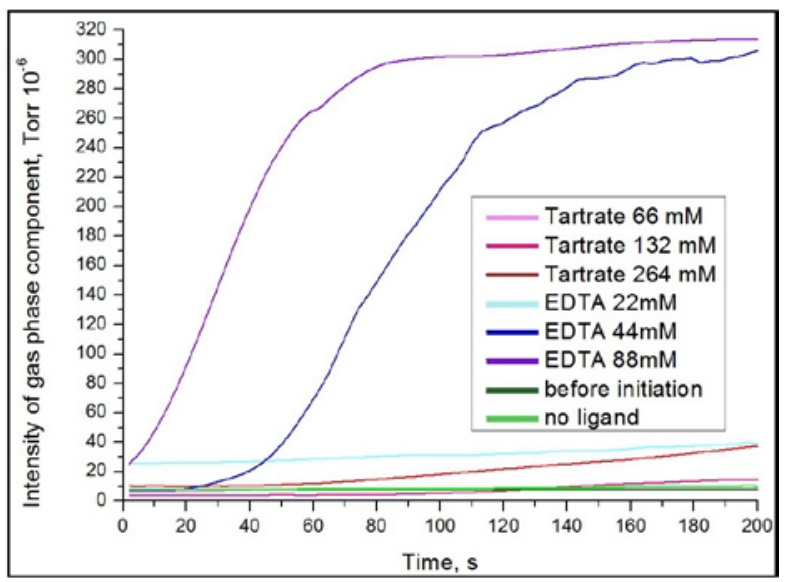

Fig. 3 The changes in time of the intensities of gas phase products generated from solutions with different concentrations of ligands.

The study of the changes in time of the composition of the gas phase components during laser deposition process for all studied solutions demonstrates a direct dependence on the presence of laser irradiation. Thus, the laser light initiates the gas phase generation within the laser beam focus. When laser is off this process stops and the system relaxes to its original state (Fig. 4). The latter issue supports the idea that the catalytic activity occurs only during the laser generation of metal copper particles.

The series of experiments on the catalytic reforming of ethanol in an aqueous medium in the presence of aqueous copper chloride demonstrate the simultaneous change of the products of complete and incomplete catalytic reforming [14] (hydrogen, ethanal, ethylene and methane) when laser is on and off (Fig. 5).

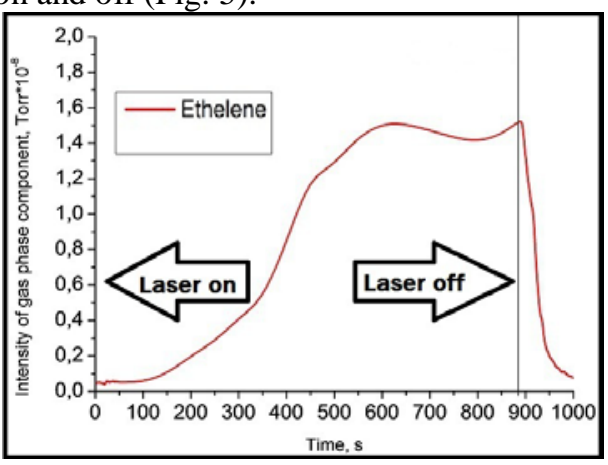

Fig. 4 The changes in time of the intensities of ethelene generated from solutions 2 .

For the first time we were able to conduct the LCLD process in non-aqueous environment. The structures deposited from copper oleate in hexane exhibit low electrical resistance.

$$
\mathrm{CH}_{3}-\mathrm{CH}_{2}-\mathrm{CH}_{2}-\mathrm{CH}_{2}-\mathrm{CH}_{2}-\mathrm{CH}_{3} \rightarrow \mathrm{CH}_{2}-\mathrm{CH}=\mathrm{CH}-\mathrm{CH}_{2}-\mathrm{CH}_{2}-\mathrm{CH}_{3}+\mathrm{H}_{2}
$$

Gas phase reaction products of solution of copper oleate in hexane showed a simultaneous change of the amount of the gas phase products (hydrogen and hexene) when laser is on and off (Fig. 6). The calculated conversion of 
hexane to hexene in solution after deposition and distillation is up to $4.48 \%$.

The solution 2 containing EDTA was analyzed by NMR spectroscopy before and after laser irradiation. The proton NMR spectra of this solution are presented in Fig. 7.

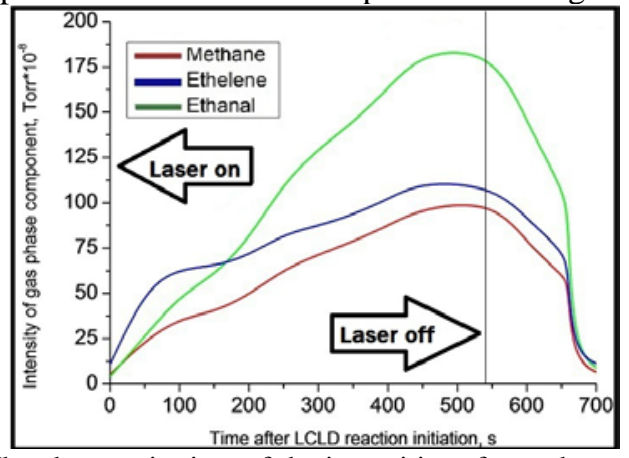

Fig. 5 The changes in time of the intensities of gas phase products generated from solution containing $\mathrm{CuCl}_{2}\left(0.02 \mathrm{~mol} \mathrm{~L}^{-1}\right)$ and ethanol.
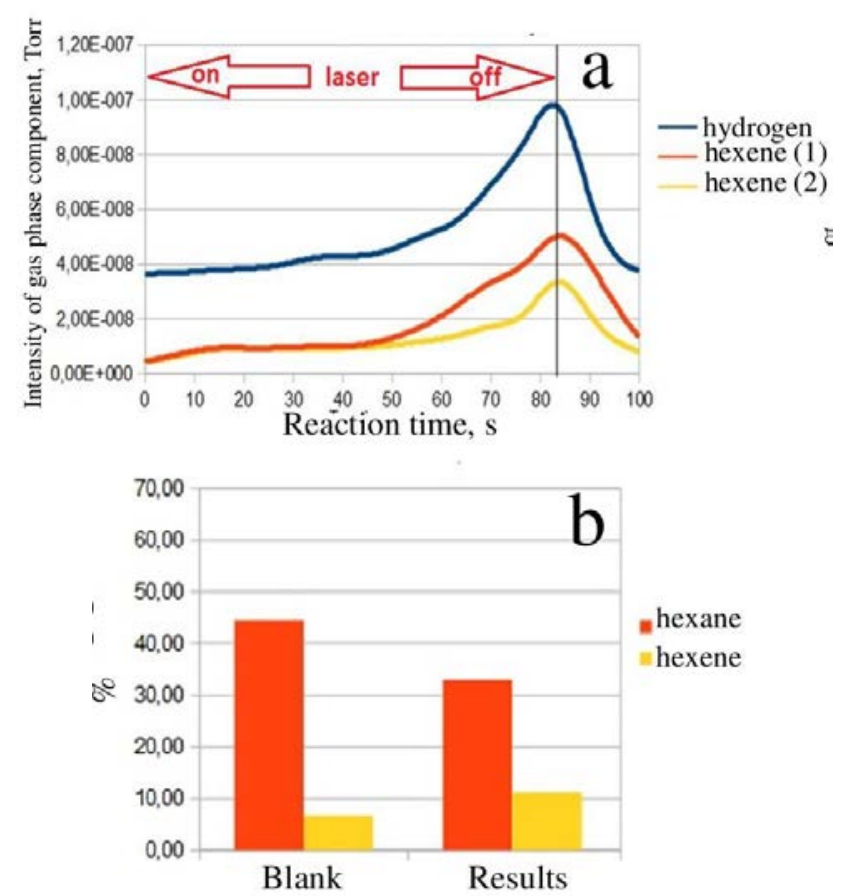

Fig. 6 (a) The changes in time of the intensities of gas phase products generated from solution containing copper oleate $(0.02$ mol $\mathrm{L}^{-1}$ ) in hexane. (b) The conversion of solution components, where (Blank) is initial solution composition and (Results) is solution composition obtained after LCLD reaction and distillation from solution containing copper oleate.

The signal of carboxylic protons (Fig. 7 red H) disappears after laser irradiation which indicates that the decarboxylation reaction occurred. The $1.07 \mathrm{ppm}$ signal was attributed to $\mathrm{CH}_{3}$ group of the primary amine formed during the laser irradiation. Also the lost of signal corresponding to methylene protons located in alpha position to the carboxyl group was observed after irradiation.

We proposed the possible mechanism of EDTA decomposition and formation of ethylene during catalyzed LCLD process (Fig. 7). In the first step, the decarboxylation of the carboxyl groups of EDTA is followed by formation of carbanion upon laser irradiation. In the second step, the intramolecular nucleophilic attack of the carbanion with subse- quent introduction of methylene between nitrogen and carbon atoms is followed by formation of an amine derivative of propanoic acid. In the third step, the decarboxylation of a carboxyl group of amine derivative of propanoic acid occurred which results in generation of carbanion. Finally, the unstable intermediate with a negative charge on nitrogen atom forming during the cleavage of ethylene splits off a proton from the solvent molecule (water).

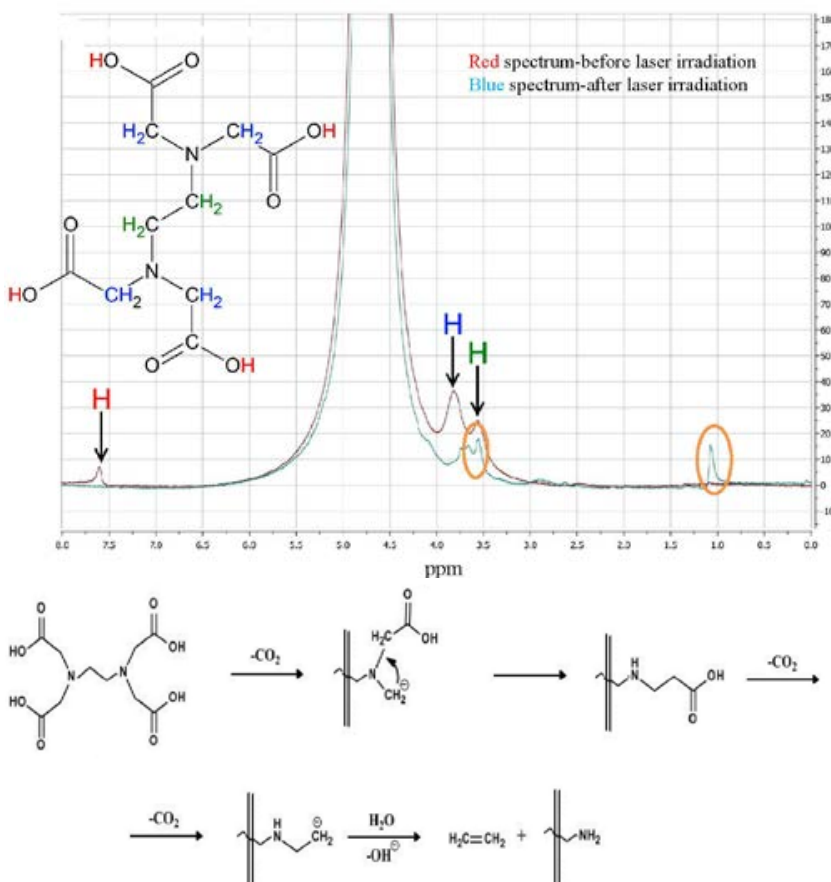

Fig. 7 The proton NMR spectra of solution 2 recorded before and after laser irradiation and the scheme of possible mechanism of EDTA decomposition and formation of ethylene during catalyzed LCLD process.

\section{Conclusions.}

The continuous laser generation of metal catalysts for organic synthesis reactions through the introduction of soluble metal salts directly in the reaction mixture was performed. The organic components contained in the deposition solution (phenol-formaldehyde resin, EDTA, K-Na tartrate, sorbitol, etc.) may act as reducing agents during the process of continuous laser metal catalyst generation. The catalysis of the studied organic reactions by laserinduced catalysts occurs exclusively during the laser irradiation of the reaction mixture. In contrast, the copper structures deposited by the LCLD method exhibit no catalytic activity. The opportunity to conduct organic synthesis reactions using in situ generation of metal copper catalysts was shown for the next model reactions: catalytic reforming of alcohols in aqueous solution and catalytic dehydrogenation of alkanes. The possible mechanisms of the product formation during laser-induced decomposition in these reactions were proposed. Thus, the approach used in this work allows to carry out selective reactions of organic synthesis using simple soluble metal salts instead of expensive and hardly accessible metal catalysts and demonstrates a huge potential for further applications in catalysis.

\section{Acknowledgments.}

I.A.B., L.S.L. and D.I.G. acknowledge the Russian Fund for Basic Research (grant № 16-03-00436). V.A.K. and 
I.I.T. acknowledge Saint Petersburg State University for a research grants (2015-2017, 12.38.219.2015). The authors also express their gratitude to the SPbSU Nanotechnology Interdisciplinary Centre, Centre for Optical and Laser Materials Research, Chemical Analysis and Materials Research Centre and Magnetic Resonance Research Centre.

\section{References.}

[1] V. Kochemirovsky, S. Fateev, L. Logunov, I. Tumkin, S. Safonov: Int. J. Electrochem. Sci., 9, (2014) 644.

[2] V. Kochemirovsky, E. Khairullina, S. Safonov, L. Logunov, I. Tumkin, L. Menchikov: Appl. Surf. Sci., 280, (2013) 494.

[3] M. Panov, I. Tumkin, A. Smikhovskaia, E. Khairullina, D. Gordeychuk, V. Kochemirovsky: Microelectron. Eng, 157, (2016) 13.

[4] V. Kochemirovsky, M. Skripkin, Yu Tveryanovich, A. Mereshchenko, A. Gorbunov, M. Panov, I. Tumkin, S. Safonov: Russ. Chem. Rev., 84, (2015) 1059.

[5] K. Kordas, J. Bekesi, R. Vajtai, L. Nanai, S. Leppavuori, A. Uusimaki A, K. Bali, T. George, G. Galbacs, F. Ignacz, P. Moilanen: Appl. Surf. Sci., 172, (2001) 178.

[6] D. Kim, C. Choi: J. Nanosci. Nanotechnol., 13, (2013) 7581.

[7] O. Lozhkina, M. Panov, L. Logunov, I. Tumkin, D. Gordeychuk, V. Kochemirovsky: Int. J. Electrochem. Sci., 10, (2015) 6084.

[8] M. Panov, I. Tumkin, A. Smikhovskaia, E. Khairullina, D. Gordeychuk, V. Kochemirovsky: Microelectron. Eng., 157, (2016) 13.

[9] X. Peng, K. Jiang: Adv. Mater. Res., 765, (2012) 424.

[10] G. Shafeev: Adv. Mater. Optics Electron. 2, (1993) 183.

[11]I. Tumkin, V. Kochemirovsky, M. Bal'makov, S. Safonov, E. Zhigley, L. Logunov, E. Shishkova: Surf. Coat. Tech., 264, (2015) 187.

[12] H. Yokoyama, S Kishida, K. Washio: Appl. Phys. Lett., 44, (1984) 755.

[13] Yu. Tver'yanovich, A. Kuzmin, L. Menchikov, V. Kochemirovsky, S. Safonov, I. Tumkin, A. Povolotsky, A. Manshina: Mendeleev Commun., 21, (2011) 34.

[14] S. Schmidt, S. Tanielyan, N. Marin, G. Alvez, R. Augustine: Topics in Catalysis, 53, (2010) 1214.

(Received: June 20, 2016, Accepted: May 7, 2017) 